\title{
A new insight to the physical interpretation of activated carbon and iron doped carbon material: sorption affinity towards organic dye
}

\begin{abstract}
To enhance the potential of activated carbon (AC), iron incorporation into the AC surface was examined in the present investigations. Iron doped activated carbon (FeAC) material was synthesized and characterized by using surface area analysis, energy dispersive X-ray (EDX), temperature programmed reduction (TPR) and temperature programmed desorption (TPD). The surface area of FeAC $(543 \mathrm{~m} 2 / \mathrm{g})$ was found to be lower than AC $(1043 \mathrm{~m} 2 / \mathrm{g})$ as a result of the pores widening due to diffusion of iron particles into the porous AC. Iron uploading on AC surface was confirmed through EDX analysis, showing up to 13.75 wt.\% iron on FeAC surface. TPR and TPD profiles revealed the presence of more active sites on FeAC surface. FeAC have shown up to $98 \%$ methylene blue (MB) removal from the aqueous media. Thermodynamic parameters indicated the spontaneous and exothermic nature of the sorption processes.
\end{abstract}

Keyword: Iron impregnation; Enhanced potential; Physisorption; Exothermic 